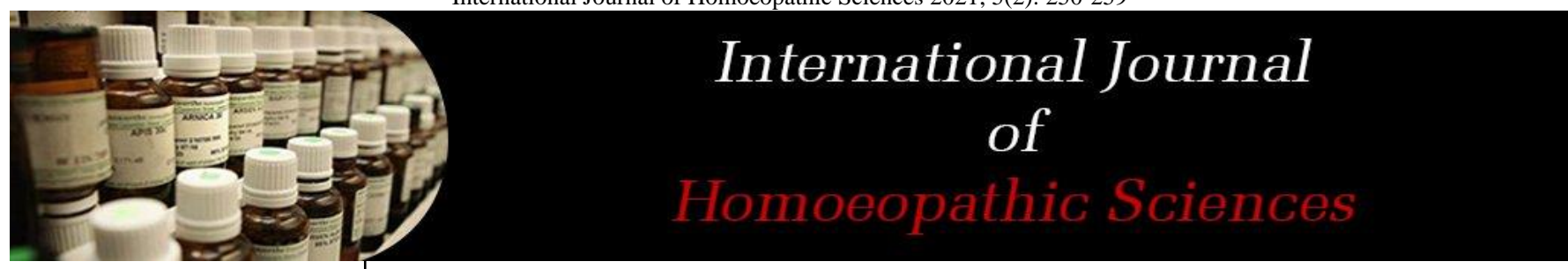

E-ISSN: 2616-4493 P-ISSN: 2616-4485 www.homoeopathicjournal.com IJHS 2021; 5(2): 250-259

Received: 28-01-2021

Accepted: 30-03-2021

Dr. Sudhansu Sekhar Moharana

Professor and Head, Department of Repertory, Yenepoya Homoeopathic Medical College and Hospital, Mangalore, Karnataka, India

Corresponding Author: Dr. Sudhansu Sekhar Moharana

Professor and Head, Department of Repertory, Yenepoya Homoeopathic Medical College and Hospital, Mangalore, Karnataka, India

\section{Psora theory of Hahnemann is scientifically immunological sensitization or psycho - pathological somatization or both?: The scientific study with case analysis}

\author{
Dr. Sudhansu Sekhar Moharana
}

DOI: https://doi.org/10.33545/26164485.2021.v5.i2d.392

\begin{abstract}
Master Hahnemann's Psora theory has been tested and has undergone the acceptance in obstinate cases where suppression of skin diseases found and has giving remarkable success. The criticism (antithesis) in case of failure in scabies and scabies with superadded fungal or bacterial infection amongst the practicing homoeopaths. Dr. Burnette, Dr. Dugeon did not accepted that scabies can be cured by internal medication of Sulphur alone experienced through their practice. Dr. Clarke opined that 'all the diseases cannot be categorized into three broad categories only. We need further classification like Burnette's vaccinosis'. Hebbert A. Roberts called nutritional deficiency and functional disorders as Psora.

As Truth is the daughter of time, not of any authority (Lord Bacon), so also psora theory undergoes the scientific processing to verify its validity. In this article, by the help of pathology and psychopathology psora theory is analyzed and interpreted. The pathogenesis of allergic and autoimmune disease giving rise to psoric manifestation is illustrated in figure $1 \& 2$. The justification of using scabicidal natural or artificial lotion or soap application in scabies along with internal medication as stated by Dr. Dugeon and Dr. Burnette is proven with scientific reasoning.

Lastly practical utility of Psora theory for prescription with case studies as per scientific approach is thoroughly elaborated. Prevention of skin diseases by hygiene maintenances and protections from psoric manifestation by simultaneous internal intake of antipsoric medicine as per totality and cleanliness of skin diseases and in case of scabies additional external scabicidal application and/or cleanliness with scabicidal soap are vividly illustrated.
\end{abstract}

Keywords: psora, sensitization, somatization, suppression, pathology, psychopathology

\section{Introduction}

'After 12 long years of practice, Master Hahnemann noticed that in spite of best suited medicine administered as per totality some of the patients either were not responded to treatment at all or remained in half cured. Master's analytical mind could able to discover that in almost all cases where medicine either not responding or patient is in half cured, there was suppression of non - venereal or venereal skin diseases. He gave the name Psora to the crisis occurring after suppression of non - venereal skin diseases' ${ }^{[1]}$ and named Syphilis to crisis occurring after suppression of chancre of Syphilis and Sycosis to the crisis occurring after suppression of buboes of gonorrhea. The suppression by external application with sulphur for non - venereal skin diseases, with mercury for external use in chancre of syphilis, and with silver nitrate for external use in gonorrheal warts or buboes etc. leads to mostly three broad categories of chronic diseases, these were psora, syphilis and sycosis respectively.

\section{Evolution}

'Master Hahnemann observed that the dyscrasia such as bronchial asthma, allergic rhinitis, epilepsy etc. caused by suppression of non - venereal skin diseases by external application' ${ }^{[2]}$. He also traced its origin and found that in ancient time the skin disease most prevalent was Leprosy but was unable to trace its origin. When Master was not able to trace its origin, then by the help of theology given in Bible he described it existed from the day one when the Adam and Eve took the forbidden apple. No matter, itch dyscrasia existed, caused numerous diseases by suppression of skin diseases through external applications by sulphur or mercury 
or any other external application. Master Hahnemann says, "Gradually I discovered more effective means against this original malady that caused so many complaints; against this malady which may be called by general name of Psora i.e. the internal itch disease with or without its attendant eruption on the skin. so also frequent epistaxis, the accumulation of blood in veins of rectum and the anus, discharge of the blood from the same (blind or flowing piles), haemoptysis, haematemesis, haematuria, and deficient as well as too frequent menstrual discharges, night sweats of several years duration, pachamnt like dryness of the skin, diarrhoea of many years standing, as well as permanent constipation, and difficult evacuation of the bowels, long continued erectic pains, convulsions occurring repeatedly for a number of years in short thousands of tedious ailments of humanity called by pathology with various names are with few exceptions true descendant of this many - formed Psora alone" ${ }^{[1]}$.

Psora as causes various diseases in different personalities When Hahnemann surveyed he found Ludwig Chirstian Juncker in his Dissertatio ex Scabie Repulsa, Halle, 1750, p. $15-18$, that "with young people of Sanguine temperament the suppression of itch is followed by phthisis, and with persons in general who are of a sanguine temperament it is followed by piles, haemorrhoidal colic, and renal gravel; with persons of sanguine-choleric temperament by swelling of the inguinal glands, stiffening of the joints, and malignant ulcers (called German Todenbruche); with fat persons by a suffocating catarrh, and mucous consumption; also by inflammatory fever, acute pleurisy, and inflammation of the lungs. He further states that in autopsies the lungs have been found indurated and full of cysts containing pus; also other indurations, swellings of the bones and ulcers have been seen to follow the suppression of an eruption. Phlegmatic persons in consequence of such suppression suffered chiefly from dropsy; her manse were delayed, and the when the itch was driven away during their flow, they were changed into a monthly haemoptysis. Persons inclined to melancholy were sometimes made insane by such repression; if they were pregnant the foetus was usually killed. Sometimes the suppression of the itch causes sterility, in nursing women the milk is generally lacking, the menses disappear permanently; in older women the uterus become ulcerated, attended with deep, burning pains, with wasting away (Cancer of the womb)" ${ }^{[2]}$.

\section{Post Hahnemannian concept regarding psora}

Psora as per Dr. Kent: 'The itch is looked upon as a disgraceful affair; so is everything that has similar correspondence; because the itch in itself has a correspondence with adultery, only one is adultery as to internal and the other two externals, one succeed other' ${ }^{[3]}$.

Psora as per Dr. Robert A. Herbert: Psoric miasmatic disorders: All Functional Disturbances and Nutritional Disturbances ${ }^{[4]}$.

Sycotic miasmatic disorders: Overgrowth and extra deposits [5].

Syphilitic miasmatic disorders: Cardinal signs of syphilitic taint is destruction of tissues ${ }^{[6]}$.

So the mind of Psora is active, Sycosis mind is malactive and Syphilis mind is inactive.

\section{So pathology also categorized to three phases}

Growth/tumor: Psoric miasm growth: Regressible e.g. Warts, uricarial rash/eruptions.

Sycotic miasm growth: Non - regressible e.g. Tumors of both benign \& Malignant.

Syphilitic miasm growth: Non - regressible e.g. Cancers.

Ulcers: Psoric miasm ulcer: Reversible e.g. Acute peptic ulcer. Superficial ulcer.

Sycotic miasm ulcer: Irreversible e.g. Cancers.

Syphilitic miasm ulcer: Irreversible e.g. Guma, indolent painless ulcer (leprosy), \& cancer ulcers.

\section{Diseases and pathological conditions ascribed for three miasms psora, sycosis and syphilis}

- Psora: Functional, reversible and nutritional deficiency disorders such as urticarial rash, acute peptic ulcer, arthralgia, acute pyogenic inflammations of skin, anaemia, hypoprotienaemia, kwasiorker, marasmus.

- Sycosis: Proliferative, odematous and defective growth disorders such as uric acid diathesis, rheumatic diathesis, tumor tendency e.g. Von Recklinghausen's disease.

- Syphilis: Degenerative disorders with inactivity such as haemorrhagic tendency, ulcer tendency, atrophy of glands, Pre-mature old age, ulcerative cancers etc.

\section{Kentian concept regarding psora}

As and when the divine aura descends from heaven, it first influence soul, then influence the mind and lastly influence to body, so also when a psycho-social pollutant enters, it first affects soul (vital force/vital principle), then to mind and lastly affects to body. So it is the mind where the affection is learned first in the form of Mental itch, 'the itch is looked upon as a disgraceful affair; so is everything that has similar correspondence; because the itch in itself has a correspondence with adultery, only one is adultery as to internal and the other to externals, one succeed other'. In case of ill thought, ill action and ill reaction because God wants us to live happily in His beautiful world by thinking good things, doing nice things and also reacting in a positive benevolent manner. Dr. Kent says, "Thinking, willing, and doing are the three things in life from which finally precede the chronic miasms". The mental itch is produced while thinking ill of others due to conflict of Exterior of the mind and Interior of the mind. So as per Dr. Kent's version it is "As long as man continued to think that which was true \& held that which was good to the neighbor that which was uprightedness \& Justice so long as man remains in the earth free from the susceptibility to the disease, because that was the state in which he was created" [7]. The Exterior of the mind is enriched with all the selfish instincts whereas the Interior of the mind is endowed with the conscience (the judgment power of right and wrong), permitting only for doing right things. So when the wrong thoughts are not eradicated by the command of Interior of the mind, pollutes the whole economy of the person. As a result of that pollution, the itch is reflected in mental and physical state before any disease occurrence known as Latent Psora. This is one of the reason for which man is known from his mind, which serves as major criteria for his personality or portrait. In this regard, Dr. Kent philosophy thus explains that Man is prior to the organs. Man is the will and understanding and the house which he lives in his body. So, as per Dr. Kent qualified mental generals and then physical generals are of 
prime importance both in miasmatic analysis and building portrait of the disease as per the Kent's version. Everything is harmoniously working in well man, consider the man, heal the sick ${ }^{[8]}$.

\section{Concept of psychosomatic diseases correlating Kent's concept of psora}

This is the concept of modern science laid down by greatest psychoanalyst Dr. Sigmund Freud. He devised the mind into three imaginary categories e.g. Id, Ego, and Superego ${ }^{[9]}$. The Id is designated as our primitive pleasure guided by instinct deals with pleasure principle, Ego is assigned to our reasoning and social recognition pleasure for social acceptance deal with reality principle and superego is the conscience for supreme achievement for good things only deal with moral. The differential examples of these three concepts - Id, Ego and Superego are:

1. To have a desire of marriage by mere sight of a beautiful girl is the Id.

2. To have a marriage proposal with the chosen girl after financial and social settlement is the Ego.

3. To wed only to the girl finding in her capability of helpful personality, research ability and attitude in sharing and caring of your every work, respectful manner to in-laws family and elders essentially constitute the Superego where one lives for others for better achievement and novel purposes. Marriage of Marrie Scoladvaska and Professor Perrie Curie is the ideal superego when both share and care each other's scientific talent and did everything together and lastly for their work they were honored with noble prize.

\section{Co - relation with homoeopathic concept of psora}

Dr. Sigmund Freud said the same thing what Dr. J.T. Kent told i.e., man suffers from the both physical and mental diseases, when there is a tension due to conflict. Dr. Sigmund Freud told it as the conflict of Id and Ego or Id with Superego mainly through approach-negative conflicts, sometimes through approach-approach conflicts. To get rid of the tension due to the conflict of the Id and Ego or Id and Superego he devised various defense mechanisms out of which sublimation is the high novel thinking with positive attitude is the best, rationalisation (the denial, telling not good for me) is the better and regression (forgetting the bitter past) is the good but escape from bitter truth by intoxication is worst defense, fantasy (day dreaming) is the worse and suppression by displacement is the bad one. If man cannot relief his tension by good defense mechanisms like sublimation or rationalisation or regression and compelled to suppress, he dreams his desire things in sleep and fulfills his instincts by fruitful dreams or sometimes the obstacle comes in his way visualizes through dreams in form of ghosts, dreadful animals or his man in failure etc. Thus dreams reveal the inner desire of man even if it is in subconscious level. When man develops tension due to failure of all these defense mechanisms and dreaming he develops mental diseases in form of Neurosis or Psychosis or Physical diseases of various forms called as psychosomatic diseases. Now - a - days, in modern competitive society psychosomatic diseases are more common amongst intellectuals for which the psychiatrist and doctors trying for assessment of mental state and trait (internalized mental state) with causation for treatment of their psychosomatic diseases.

\section{The scientific interpretation of concept of psora}

As per the theory of Dr. S. Hahnemann, the Psora is the mother of all the non-venereal diseases being suppressed with external applications. As per the theory of Dr. J.T. Kent, Psora penetrates at first in the psychic plane, itching the mental state and then pollute the whole systems of organisms i.e. the vital principle is deranged. Thus to comprehend the evolution of Psora explained by the stallwarts of homoeopathic faculty in the light of modern medical science it is necessary to learn the neuro-endocrinoimmunological balance maintained in our body. The neurological balance is responsible for the healthy behavior and sound mental activity whereas the optimum endocrine activity is responsible for growth and metabolism of the body. Then the optimum immunological activity is for defense against the external noxious morbific agents that produce the disease.

These three neuro-endocrino-immunity machineries of our body together harmoniously co-regulate each other's activity for maintenance of balance and there by sound health ensues. How mental stress is responsible for development of Psora is illustrated in the following Fig.1 explaining the penetration of Kentian itch at the mental plain.

From Fig.1, it is understood that if the emotional stress is sustained one, it causes the various types of psychosomatic ailments which includes emotional phenomena like flushing, palpitation, Diarrhoea etc., physical diseases like peptic ulcer, hypertension, convulsion and allergic diseases like bronchial asthma, eczema, urticaria and ultimately susceptibility to various form of infectious diseases due to the neuro-immunological imbalance leading to exaggeration activity of one or two machineries and consequently weakness of the others. This indicates the scientific explanation of Kentian theory of Psora, itching first in mind and polluting the whole system leading to origin of psychosomatic diseases.

\section{Psora as immunological sensitization}

Similarly, if the stress is biological i.e. due to bacteria, viruses, fungi, parasites, their occur inflammations so as to localize and eliminate the causative agent through activation of body's cellular and humoral immunity. As a result this causative agents like bacteria, viruses etc. are killed and eliminated from our body. But in case of infection of scabies (gravid scabies which is up to 0.3 milimeter) ${ }^{[10]}$, the immunity is not sufficient enough to eradicate them by inflammatory response without the help of external application of medicine because the size of macrophase the largest scavenger cell is $(12-18 \mu$ in diameter). If those causative factors are killed by external applications then the effects (eruptions, itching etc. due to superadded infection) there of subside as generally done in allopathic medicines. For example, "The itching and rash associated with scabies derive from a sensitization reaction directed against the excreta that the mite deposits in its burrow. An initial infestion remains asymptomatic for up to six weeks, and a re-infestation produces a hypersensitivity reaction without delay. Burrows become surrounded by infiltrates of eosinophils, lymphocytes, and histiocytes, and a generalized hypersensitivity rash later develops in remote sites" [11]. But in some cases when no treatment is given from beginning due to prolong irritation of the scabies or ringworms and even in impetigo the organism sensitizes the skin so that the 
allergic phenomena (eczema etc.) are produced. Once there is onset of allergic phenomenon it never remains as local ailment as it before and transforms to the general ailment of whole of the organism. Thus the inflammation cycle is repeated unless they are cured with the Hahnemannian method of antipsoric treatment and give rise to Sycotic miasmatic state by formation and proliferation of grannuloma and thickening of epithelium explained detail in Fig. 2.

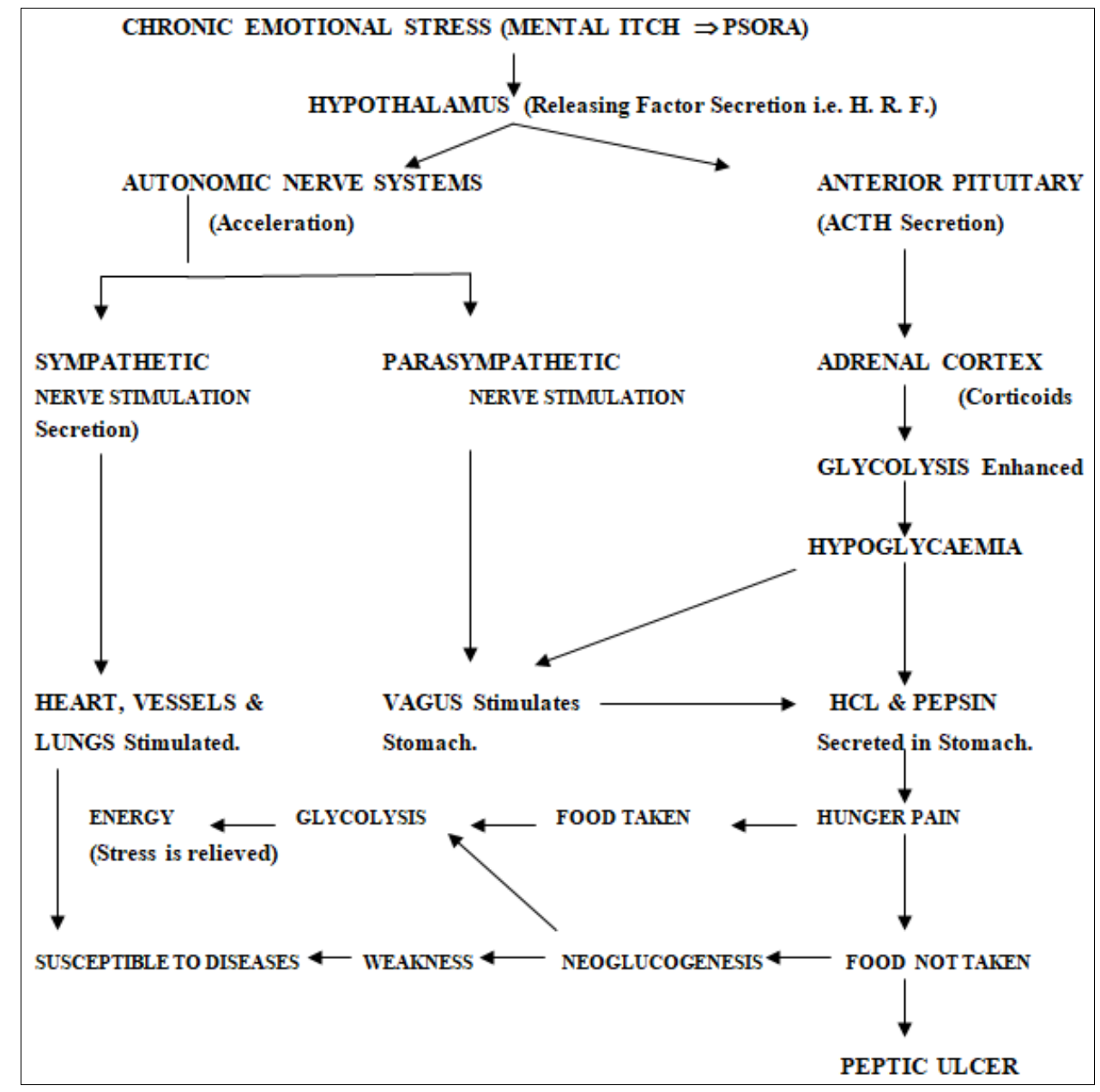

Fig 1: Illustration of how mental stress is responsible for development of psora

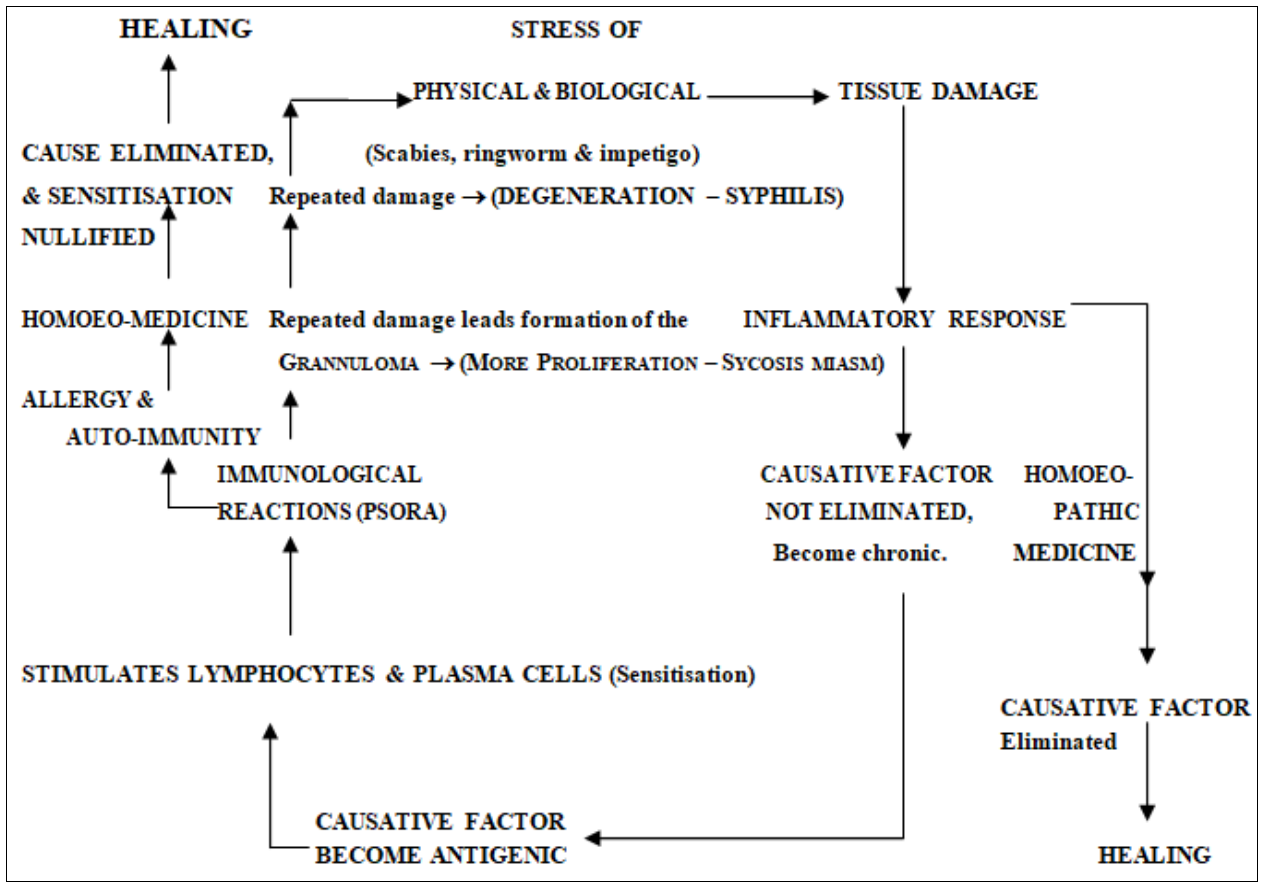

Fig 2: Formation and proliferation of grannuloma and thickening of epithelium 
If the local symptoms of those general ailment, i.e. allergic phenomenon is annihilated by external application then it is obvious that there will be reappearance of same allergic manifestation at some weaker site. And at the same time it is also naked truth that if Scabies are simply treated homoeopathically by internal medicines alone without giving importance of external applications and special scabicidal method of cleanliness so as to avoid re-infection, it is impossible to cure the patient permanently. This is from the scientific knowledge of pathology and microbiology that the both cellular and humeral immunity of our body is not strong enough to such an extent to kill the mite which is up to $0.3 \mathrm{~mm}$ in length i.e. 300 micrometer (ordinarily visible by a magnified lens) acting persistently as maintaining cause as the diameter of Neutrophil is $9-15$ micrometer ${ }^{[14]}$, diameter of macrophase about 21 micrometer ${ }^{[15]}$, giant cells are 40-120 micrometer in diameter ${ }^{[16]}$. So treatment of scabies is just like that of eradicating the pediculosis of skin by internal intake of homoeopathic medicines. So the scabies treatment in all cases must be by external application which does not cause suppression in all the cases. The suppression only occurs when there is sensitization of part which mostly occurs in chronic untreated scabies or ringworm or pyoderma.

These are not only the deductive logic derived and corelated from the knowledge of pathology and microbiology but also from my personal experiences I have obtained in several cases. The examples of some cases are quite interesting and so cited here. The complaints of Mr. X was severe itching and eruptions for which his case - history was taken reportorized and the medicine came to appear was Graphites. I decide to give it in 50 millisimal scale because the remedy was the result of his individualization. To elaborate, the patient as chilly, aversion to sweets, meat and constipated, I had started the Graphites from LM 3 potency up to LM 23 between dt.2.3.88 to dt.18.9.88. The itching and eruptions totally vanished then appear at interval of seven to ten days which was healed up by the drainage of the exudations while was in the process of treatment. Although the indicated remedy was great antipsoric and suited best to the patient still then it could not be able to cure patient totally with strict observations of cleanliness, for more than 6 months to avoid reinfection. After that case, I did not dare to experiment in clinic but could experiment it in my family members with intake of Homoeopathic medicines and strict observation of cleanliness i.e. boiling of all cloths and bed-sheets to avoid re-infection but the same was the result. At last I was compelled to advise the family members to use scabicidal soap in bathing and using besides Homoeopathic medicines intake.

The answer is here for these cases is very simple. The itch mite is up to $0.3 \mathrm{~mm}$ (300 micrometer) to $0.4 \mathrm{~mm} \mathrm{(400}$ micrometer) in length ${ }^{[15]}$. So the female and male itch mite is so big that cannot be engulfed by phagocytic cells of body macrophase (about 21 micrometer), giant cells (40-120 micrometer in diameter). The itch mites burrow in the epidermis in irregular Zigzag shape about $3 \mathrm{~mm}$ to $15 \mathrm{~cm}$ long and lay eggs there. So simply washing with ordinary soap cannot remove them. By the internal intake of Homoeopathic medicine there can be healing of Impetigo of Pyoderma which is created by secondary streptococcal or staphylococcal infections. But the itch mite itself remains as such being immune to cellular defense and burrowing and its faecal matters in skin act as a maintaining cause of skin irritation and followed by secondary infection. This becomes evident after the discontinuity of Homoeopathic medicine for scabies. I, on enquiry of literature also found that my observation coincides with the observation of Dr. Burnette in his book "Change of life in Women" There he mentioned his failure in curing ordinary itch due to Sarcoptes Scabil infection with potentised dose of sulphur high, higher in right way and on the other hand the success of allopathic doctors with sulphur lotion, soap and water, in the chapter of "Hahnemannian Doctrine of Psora Restated". After repeated and persistent failure Dr. Burnette concluded that "I would restate the doctrine thus: You cannot cure the itch by dynamic medication, and you must therefore kill the ascarii; they should be killed in spot, the sooner the better; you cannot kill ascari with dynamic remedies, and they should be killed at once. But I am not speaking of its concomitant constitutional erruptions brought forth by ascari, neither do I say that the ascari may not poison the blood-indeed I think they do, and therefore they sulphured to death instanter. But, and this is very important, if the ascari have called forth an erruption from a previously existing internal state, THIS eruption may NOT be got rid of by external remedies." "So the ordinary itch cannot be cured by dynamic medication and the acarie should be killed on the spot, the sooner is the better" ${ }^{[12]}$. He further noted that result of suppressing the constitutional eruptions that have been called forth from their internal lurking by the Acarii themselves or by their poison that has to be feared. Similarly Dr. Dudgeon in his writing 'Lectures in Theory and Practice of Homoeopathy' in the opinion that "I have attempted many times to cure the itch according to Hahnemann's directions, but have never succeeded in the very slightest degree. The disease depending on the existence in the skin of a parasitic animal, the main indication to be attended to is the destruction of this troublesome vermin. As the habitat of this insect is limited to certain definite parts of the skin, our applications for its destruction should be limited to those parts. Experience has shown that many substances are capable of affecting its slaughter. Sulphur in the form of tincture or ointment, mercurial ointment, preparations of lead and copper, oil of terpeentine, and finally simple fatty substance and soft soap are all the capable of producing the desired result. The treatment I and others found quite successful is to ascertain, from the presence of canaliculi, where the acarus is, and in recent cases it will generally be found to be confined to the hands of the wrist; these parts are be washed once or twice a day with a mixture of flower of sulphur and spirits of wine. In a few days the animals are all destroyed, and the general eruptions will go off in a few more days. In a few more days the animals are all destroyed, and the general eruption will go off in a few more days, quicker probably if assisted by the internal administration of a few doses of sulphur, we may succeed equally well by the dirtier plan of larding the parts infested with the acarus by simple ointment, cold cream, soft soap, or cod liver oil, giving sulphur internally at the same time. Baths, cleanliness and frequent changes of linen are important adjuncts to the treatment" ${ }^{[13]}$.

\section{Scientific analysis}

After this experience, I do advise for the maintenance of cleanliness by instructing them to boil their cloths and bed sheets daily to avoid the reinfection and to wash the hands with soap and water perfectly (especially webs of fingers, 
armpits and other infected area where the mites generally borrows) and to use our external applications besides taking internal medicine. For the treatment of Tinea infections (ringworm) our Homoeopathic medicines acts very well and the sanitation of wearing daily boiled washes cloths and maintenance of cleanliness is needed to avoid re-infection. So the Hahnemannian conception of Psora being the mother of all the thousands of incredibly various acute and chronic (non-veneral) disease does not hold good in all the cases. Homoeopathic physicians very often confuse seeing the fact that if an asthmatic patient suffers from a skin disease nonallergic in nature with all the pyogenic symptom and signs, the bronchial asthma patients get relief from his sufferings for the time being.

The Scientific explanation of it, is that during the staphylococcal skin infections the body produces the antibodies (Immunoglobulins-Ig.G) against those bacteria which may act as blocking antibody ${ }^{[17]}$ against the regainic antibody (i.e. Ig.E) preventing the fall of cyclic AMP and subsequent prevention of mast cells' degrannulation. Thus the allergic inflammation in bronchial mucosa is prevented responsible for asthmatic attack, and if the asthmatic patient suffers from skin diseases of eczema, urticaria etc. of allergic manifestation, the bronchial mucosa is often relieved of allergic inflammation owing to the fact that the original allergic phenomenon has been directed to other way. So the allergy once developed in the body is not a local symptom, although it manifests locally, becomes the whole bodily dycrasia. So suppression of allergic skin diseases like eczema, urticaria etc. with external applications generally leads to bronchial asthma or allergic rhinitis or gastritis or sometimes allergic meningo-encephalitis etc. which if not cured totally is transmitted as hereditary disease of the allergic nature. Thus it proves that Hahnemann's Psora is nothing but the psoric miasmatic state of the body is that the hereditary or constitutional allergic diseases initially might have been caused by sensitization of skin by the scabiee hominis infection, or ringworms, or impetigo or by vaccines or by chemicals etc. If it is (allergic phenomena) suppressed by external application becomes directly mother of other allergic diseases and indirectly becomes the mother of other diseases of sycotic and syphilitic miasmatic states by disturbing the neuro-endocrino-immune systems harmonious balance. As a result, when there is a suppression of allergic disease and if it does not find its way to be manifested, otherwise affects the other two nervous and endocrine systems either diminishing or exaggerating their activities. Thus many other diseases of non-allergic nature are also produced.

\section{Clinical application \\ Methods}

1. Hahnemann's method of Cessenta causa, Cessat effectus: Master Hahnemann has instructed us to treat psora first, whether in single miasmatic disease (only psoric in origin) or multimiasmatic disease (Psora + Sycosis, Psora + Syphilis, Psora + Sycosis + Syphilis). In multimiasmatic disease, Master instructs us to give Hepar sulphuricum instead of Sulphur or antipsoric remedy as per totality or multimiasmatic remedy having high value in Psora. This can be achieved by referring Psora rubric from Boger's Repertory, Synthesis Repertory and Complete Repertory. If in single miasmatic disease of Psoric origin, the Graphitis is indicated as per totality as per Dr. Nash a dose of Sulphur should not be given. The Graphitis would take care of annihilating psoric miasmatic dyscrasia being high grade antipsoric remedy. Similarly if in the case Mezerium is indicated or Mercurius solubilis is indicated no need of prescribing a dose of Sulphur because Mercurius is having two values and Mezerium is having one value in rubric Psora.

Moreover, Sulphur should not be prescribed in case of suppurated eczema, psoriasis, and scabies or in pyoderma or in tuberculosis as pathophysiologically Sulphur is a great absorbant. Probably by knowing this Master Hahnemann told not to use Suphur in mixed miasmatic diseases, and to use Hepar sulph instead. In case of mixed miasmatic diseases, antipsoric remedy of very high order other than Sulphur, can be used. Hepar sulphuricum and Mercurius solubilis should not be used in high potencies as they abort the suppuration. So in case of suppurated eczema, psoriasis and pyoderma, Sulphur, Hepar sulphuricum and Mercurius solubilis should not be in high potencies.

2. The miasm can be differentiating factor at post repertorial result analysis or after totality/repertorial totality: The suppression or after effect of skin diseases and/or mental tension if gives rise to other diseases such as atopic bronchial asthma, ulcerative colitis, amenorrhoea hypothyroidism or hyperthyroidism, Addision's disease or Cushing's syndrome, then first predominate antipsoric medicine should be selected at post repertorial result analysis as per the example illustrated below. Similarly grouping of functional and nutritional deficiency diseases, suppression of skin diseases, and lastly symptoms of psora (as described in master Hahnemann's Chronic Diseases in topic psora) in the case, to Psora can also be helpful in final differentiation of remedy. For example, here is the case of benign hypertrophy of prostrate of 65 years old man. Patient is a by profession a scientist of International repute who had suppression of eczema of right leg by ointment 5 years back, he is comfortable at winter season, likes fanning, desires cold food and drinks. People used tell him philosopher due to his long beard and carelessness in dressing. $\mathrm{He}$ is very expressive in seminar and very familiar with his friends. No particular symptom except difficulty in passing urine. Three drugs comes a) Sulphur, b) Calcarea carbonicum c) Lycopodium. Phosphorous \& Calcarea carbonicum and Graphitis are eliminated out as chilly patient.

- The benign hypertrophy of prostrate is sycotic miasmatic ailment.

- Difficulty in urination due to benign hypertrophy of prostrate is also sycotic miasm symptom.

- The patient is constitutionally Sulphur \& Lycopodium also due to a) Intellectual, hot patient, extrovert, but b) pathology and particular symptom is sycotic miasmatic state.

- Then we have to find out sycotic property of both the drugs. Sycotic property of both the Sulphur \& Lycopodium is also same i.e. 2 value in synthesis repertory ${ }^{[18]}$ although sulphur is the king of antipsoric medicine having value 3 in Synthesis repertory as per the multiple bar diagram: 


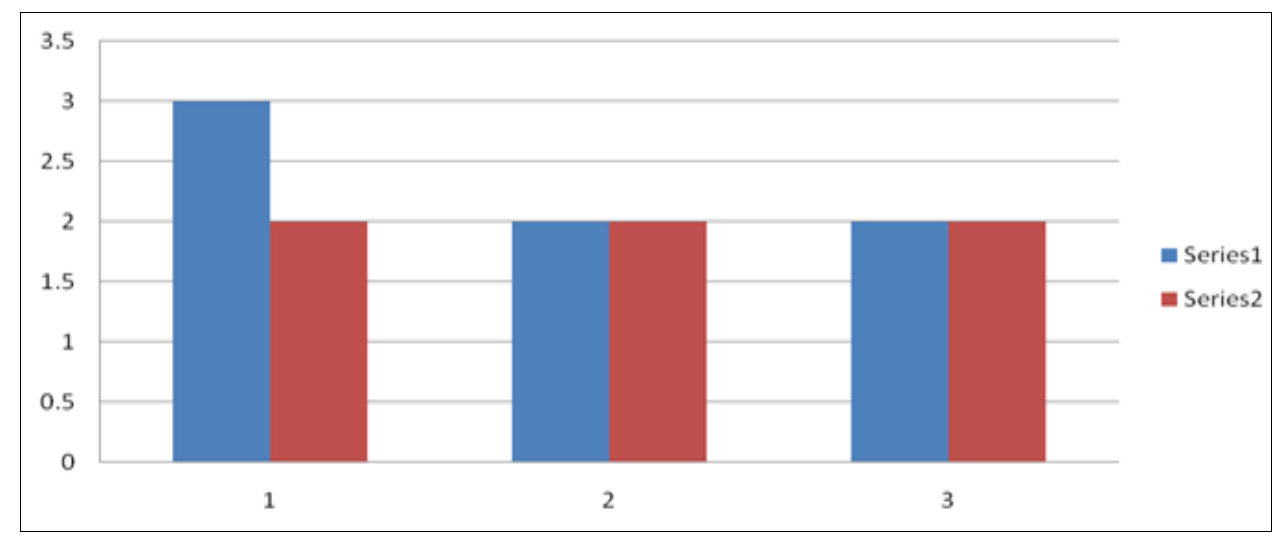

Fig 3: Sulphur (Series 1) \& lycopodium (Series 2) value as in psora (1), sycosis (2), syphilis (3)

So sulphur is the selected remedy as per the totality with suppression and for the condition of benign hypertrophy of prostrate. Lycopodium is discarded although it has same value of sycosis with that of Sulphur but patient had suppression of eczema which Sulphur had better value.

Similarly Thuja occidentalis is having value 1 in rubric Psora, 3 value in rubric sycosis and 2 value in rubric syphilis in Synthesis repertory ${ }^{[19]}$ although generally known as King of Sycosis can be ploted as bar diagram as follows for better illustration:

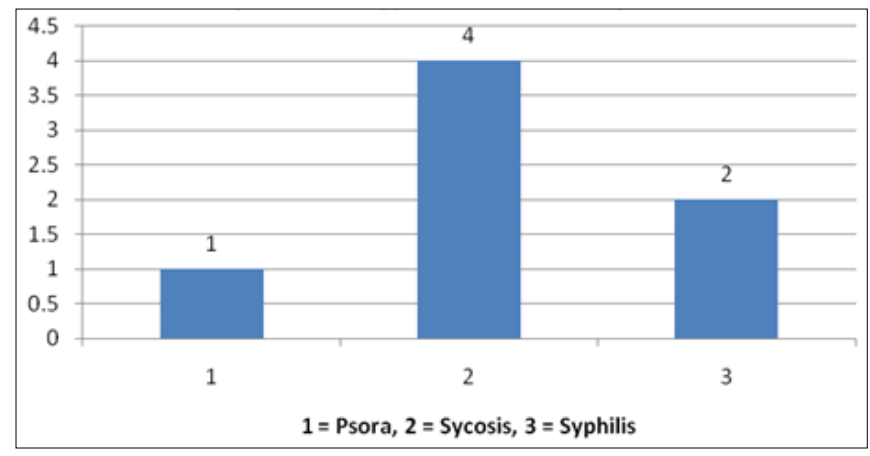

Fig 4: Psora, syosis and syphilis value of thuja occidentalis

Similarly Mercurius solubilis is known as King of Syphilis but it has value 2 in the rubric of Psora, 1 value in Sycosis and 4 value in rubric Syphilis in Synthesis repertory ${ }^{[20]}$ can be ploted as bar diagram as follows for better illustration:

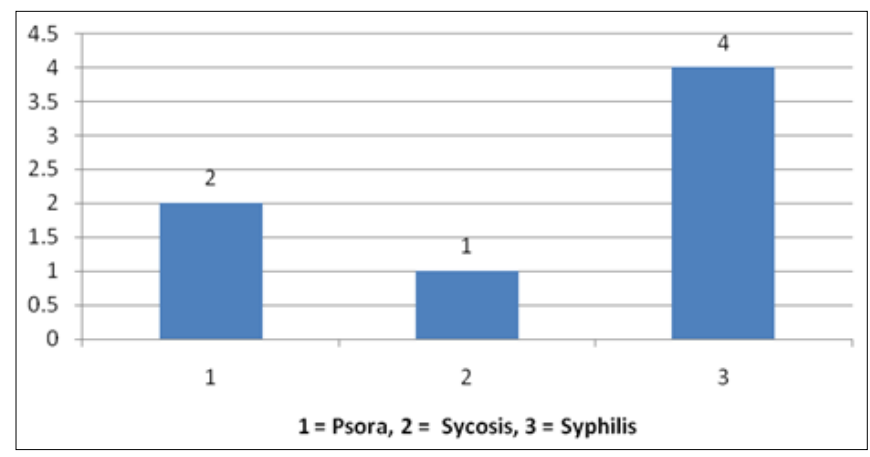

Fig 5: Psora, sycosis \& syphilis value of mercurius

Thus the King of Psora Sulphur can be used in sycosis and sycotic miasmatic state and syphilis and syphilitic miasmatic state, King of Sycosis Thuja occidentalis can also be used for Psora and psoric miasmatic state and Syphilis and syphilitic miasmatic state and King of Syphilis Mercurius can be used for Psora and psoric miasmatic state,
Sycosis and sycotic miasmatic state in the same manner as illustrated above in multiple bar diagram of Sulphur, Thuja occidentalis and Mercurius solubilis.

Some of the cases are illustrated for considering Psora in prescribing totality as follows:

Case no. 1: Mrs. 'A' aged about 35 years came to my clinic with griping pain in stomach and indigestion. I gave her Nux vomica 30C every 4 hourly in water doses basing upon the symptoms she presented. One dose I had given to her mouth at clinic and advised her to take rest for five minutes in the sofa. After five minutes she got relief and went to home taking the rest of medicines. After few hours when I was just going to close my clinic she came to me with a hurry and told me see doctor, how your medicine has caused skin rashes over my whole body. I have never known that Nux vomica causes skin rashes in either treatment or in proving. I enquired her about the food she had taken basing upon the fact that stomach has the power of avoiding digestion and interrupting absorption by reacting it to noxious food substances for which gripping pain, flatulence etc. occurs and bad food is eliminated with the help of vomiting and Diarrhoea as it so happens in food poisoning. She told me of taking wet rice and dry fish. I confirmed from her answer that she is allergic patient for which stomach reacted to allergic diets such as dry fish and when the digestion and absorption was forced by the Nux vomica 30C the allergic reaction manifested in skin causing urticaria all over body. I immediately gave her some doses of Arsenic album 30C every 4 hourly in water doses basing upon the causation that it neutralize the bad effect of animal food, then the patient was all right. This example indicates the linear directive evidence of Psora being cause of similar disease.

Case no. 2: There is another case showing the diagonal directive evidence of Psora causing the dissimilar diseases by deranging the hormonal harmony, is the case of Miss ' $\mathrm{B}$ ' of 18 years age who was suffering from primary amenorrhea. On examination of her symptoms on date 10.10.88 it was found she did not have cryptomenorrhoea. Her hemoglobin count was normal. She was fatty, she was constipated and had aversion to meat, sweet, chilly and hot demarcation in her case was quite confusing so that it was eliminated. I gave her Graphitis LM 5 once daily the eczema reappeared just after two days. Then I delayed the intake of medicine with greater intervals i.e. Graphitis LM 5 to be taken after two days intervals. After about five days the eczema was cured but there was no progress in menarche 
for about three months, the patient only reported that she was becoming slim day to day. I told her guardians that it is good sign of hormonal activity and very soon she would have menarche. Just after completion of three months when patient was taking Graphitis LM 9 in two days intervals she had menarche.

Case no. 3: There is another case showing the diagonal directive evidence of Psora causing the dissimilar diseases by deranging the immune status. A lady ' $C$ ' of 23 years age had very obstinate cough with two big tonsils enlarged came to Ahinsa clinic on dt.7.8.93 was given Belladonna 30C, every 4 hourly basing on her burning sore throat, cough complaints < by cold, fatty constitution and enlarged tonsils. When she was all right of the obstinate cough, the eczema appeared on her legs. So on dt. 15.8.93. She was then given Tuberculinum 200C, two doses, once daily in 7 days intervals. Her eczema started healing and itching subsided. Then she was cured totally with Tuberculinum 1M one dose. Thus this case showed due to eczema suppression which she failed to tell in acute case taking, reappeared by the Hering Law of cure that is going from central to periphery and reverse order of appearance. The eczema could disturb the immune system by which chronic tonsillitis occurred causing the obstinate cough. So the Psoric miasm can be treated with antimiasmatic medicine covering the other symptoms, here enlarged tonsils, phlegmatic fatty constitution, easily catching cold and eczema as a whole to which Tuberculinum was the medicine.

Case no. 4: This is the case which shows the Kentian concept of Psora being the cause of psychosomatic resultant. I had a case of sincere professional ' $D$ ' aged 52 years who had dry eczema on both legs itching often brought by one of my allopathic doctor friend to Ahinsa clinic. While taking case I had asked about any mental tension. My doctor friend told me "yes he has lot of mental tension in his profession. I also found that he was extremely laborious, keeps everything in order and when finds anything disorderliness he gets annoyed. Then he was given Nux vomica 200C one dose at bedtime on 12.11.98. After 20 days, his eczema was cured totally.

Case no. 5: This is the another similar case which shows the Kentian concept of Psora being the cause of psychosomatic ailment. My own cousin ' $E$ ' had neurodermatitis on neck during his post Graduate examination period. That time he was 23 years old. I gave him Kalium phosphoricum 30C, thrice daily with water doses basing on the tension of examination causing immunological system imbalance. He was cured of the neurodermatitis totally within seven days.

Case no. 6: Psychosomatic hypertension cases - Many cases of Hypertension which had definitely anxiety or psycho-social tension as the cause respond pretty well to treatment as per the cause and the process of somatisation there of: I had cured many cases of essential hypertension where the mental tension in job and social relationship became the cause itching mind first like that of Dr. Kent's Mental itch caused by Psora. When mental tension being mental itch was polluting the whole economy of vital principle causing essential hypertension, I used to prescribe Kaliphos.30C thrice daily with water doses upon the disturbance of sympathetic nervous system as found in Kali phosphoricum drug picture elaborated in William Boerick's Matria Medica book. Thus cured cases of essential hypertension by Kali phosphoricum reveal the evidence that the fundamental cause mental tension. (Psora as per Kentian Concept causing mental itch) should be treated first, which is deranging sympathetic system causing essential hypertension.

\section{Discussion}

Thus the psoric miasmatic state's manifestation produced in our body due to psychic or biological stressful stimuli is the expression of hypersensitivity towards any morbific noxious agents may be allergen, auto-antibodies, or microbes or grief. Then the inflammation cycle is repeated as per the Fig. 1 in case of psychic stress and as per Fig. 2 in case of biological stress or sometimes simultaneously both the events. This leads to the proliferation of mental agonising state internalized thereby the type of behavior cultivated known as trait. Physically the proliferation of grannuloma occurs followed by the fibrous tissue proliferation in case of the continuous biological or physical stress. As a result a proliferative growth occurs in response to repeated inflammations resembling the proliferation of buboes or exudative swelling of joints of that venereal miasm gonorrhoeal sycosis. We call this condition also sycotic miasmatic state although there is no causative agent of gonorrheal sycosis. Here psoric miasm in repeated attack becomes the mother of sycotic miasmatic state's manifestation.

Then if mental stress continues the regression occurs as a defense mechanism to cope up the stress. Thus forgetfulness to bitter past events occurs. Sometimes the regression becomes acute enough causing the forgetfulness of all past events and even recent past. This condition resembles the G.P.I. of quaternary syphilis. Similarly when there is prolong mental stress, the peptic ulcer occurs due to hyperacidity which is superficial in beginning, and later on becomes deep owing to repeated psychological stress causing irreversible destruction or degeneration resembling the condition of syphilitic miasmatic state. While discussing all this scientific interpretation of above Figures and clinical co-relation of the given cases, one of my colleague told me that peptic ulcer is not belong to disease of psoric miasmatic state. It is rather belong to disease of syphilitic miasmatic state. He told it as all the functional disorders are belong to Psoric miasmatic state, proliferative disorders are belong to Sycotic miasmatic state and degenerative disorders or destructions are belong to syphilitic miasmatic state. What he told that is correct in macroscopic view but microscopic vision a superficial peptic ulcer is the result of hyperacidity and inflammation of gastric mucosa which heals up spontaneously when cause is eradicated. The ulcer and inflammation heals soon when hyperacidity is prevented. So ulcer here is superficial level which heals when cause is eradicated thus psoric in nature. When ulcer penetrates to muscle layer due to negligence of hyperacidity and pain it becomes grave one resembling syphilitic miasmatic state. Then it needs the antisyphilitic miasmatic medicines like Nitric acid, Kalium bichromium., Hydrastis, Phosphorous, Lycopodium etc. So here the Psora is the mother of Syphilitic miasm. The causation - psoric mental itch should also be treated first then the effect - syphilitic miasmatic state, i.e. the deep seated peptic ulcer should be treated with 
antisyphilitic medicines. So here antisyphilitic remedies as per sector totality can be given in low potency and the constitutional medicine taking the mental features in high potency inter-currently with an initial dose of antipsoric remedy. Dr. Hahnemann stating of treating Syphilitic miasmatic state with an initial dose of antipsoric remedy thus scientifically revealed true and Dr. Kent's concept of treating man with the qualified mental and physical generals with repertorization and making the charecteristic particulars differentiation so that general should contain particulars not vice versa, is worthful. That means on greater totality assessment after repertorization of qualified generals which is of psoric miasmatic state, the antisyphilitc sector totality also fits to that medicine by differentiation, is best one. As for example, the Lycopodium, Phosphorous etc. are the three miasmatic medicines if fits best for psoric miasmatic state through mental generals and physical generals as well as the effect deep seated peptic ulcer resembling syphilitic miasm by sector totality, they are the best suited medicines as fitting to both psychic and somatic plain of patients, and also miasmatic states simultaneously. In case of diabetes mellitus and hypothyroidism when there is auto-immune process in damaging those endocrine glands as I explained the process of allergic and autoimmune disorders due to Psora, similarly the remedies should be chosen considering psoric miasmatic state as causation and syphilitic miasmatic state as a effect altogether through totality of symptoms. As a result, the auto-immune process can be stopped by anti-psoric part of that remedy by desensitization and simultaneously stopping the degeneration process by anti-syphilitic property of that mixed miasm remedy.

\section{Conclusion}

Psora theory as observed by our Master Hahnemann and Master Kent as a consequence of suppression of skin disease and/or mental tension, thus can be correlated as immunological sensitization and psychological somatization. Thus allergy and auto - immune diseases of skin such as eczema, urticarial rash, psoriasis, lichen planus etc., and psychosomatic disorders such as neuro - dermatitis, urticarial rashes etc. can be treated for antipsoric procedure through prescribing totality or by differentiating at post repertorial result analysis/totality to get a similimum.

The suppression of eruption rubric and ailments of mental tension rubric such as ailment from grief, mortification, insult etc. as found in various repertories can be used as $1^{\text {st }}$ grade symptom in evaluation of case and can be an eliminating rubric in artistic method of repertorisation. The medicines coming as high value in those rubrics can be also considered for final differentiation at post - repertorial result analysis for best medicine selection.

Lastly Sulphur is not only the drug for antipsoric treatment. If by totality, Graphitis or Sepia or Mezerium or Mercurius or Hepar sulphuricum is indicated then that indicated medicine can be given for totality instead of giving a initial dose of Sulphur ${ }^{[21]}$. Sulphur should not be given in mixed miasmatic diseases such as Psora with Sycosis, Psora with Syphilis, Psora with Sycosis and Syphis as per master Hahnemann's instruction in his Chronic diseases for the treatment of mixed miasmatic diseases instead of Sulphur, Hepar sulphuricum low potency one dose can be given (Hepar sulphuricum in high potency aborts suppuration and a grievous condition may occur). Sulphur should not be given in suppurated skin diseases and in tuberculosis as it aborts suppuration.

If Calcarea carbonicum or Lycopodium is indicated and value of Sulphur is very near to them in reportorial totality or in conceptual image, then before giving Calcarea carbonicum or Lycopodium one dose of Sulphur can be given as Sulphur, Calcarea carbonicum and Lycopodium run in hierarchy.

Some people begin the case with Sulphur. If Sulphur is indicated in non - suppurated wound or pyoderma and non tubercular cases then begin the case with Sulphur otherwise do not begin the case with Sulphur unnecessarily.

Sulphur is a trace element and in lot of vital food it is a constituent. So Sulphur containing food like onion, carrots, redish, fruits can be taken liberally. The pungent things of onion, redish and garlic can be neutralized by cooking or keeping them in vinegar or curd for sometimes by which means the subtle action of homoeopathic medicine is not damaged.

\section{References}

1. Hahnemann S. The Chronic Diseases (Theoretical Part. B. Jain Publishers (P) Ltd., New Delhi. Reprint Edition 1996, 31 - 33.

2. Hahnemann S. The Chronic Diseases (Theoretical Part). B. Jain Publishers (P) Ltd., New Delhi. Reprint Edition 1996, 48 - 49.

3. Kent JT. Lectures on Homoeopathic Philosophy. B. Jain Publishers (P) Ltd. Reprint Edition 1997, 137.

4. Herbert A. Robert. Principles and Cure of Homoeopathy. B. Jain Publishers (P) Ltd, New Delhi, Reprint edition 2003, 186.

5. Herbert A. Robert. Principles and Cure of Homoeopathy. B. Jain Publishers (P) Ltd, New Delhi, Reprint edition 2003, 236.

6. Herbert A. Robert. Principles and Cure of Homoeopathy. B. Jain Publishers (P) Ltd, New Delhi, Reprint edition 2003, 215.

7. Kent JT. Lectures on Homoeopathic Philosophy. B. Jain Publishers (P) Ltd, Reprint Edition 1997, 134.

8. Kent JT. Kent's New Remedies, Clinical cases, Lesser Writings, Aphorisms, and Percepts. B. Jain Publishers (P) Ltd, Reprint Edition 1997, 641.

9. Ryckman RM. Theories of Personality, $5^{\text {th }}$ edition, Califernia: Brooks/Cole Publishing Company 1993, 33 - 34.

10. Richard J. Pollack, James H. Maguire, Fauci A.S., Braunwald E., Harrison's Principles of Internal Medicine, $17^{\text {th }}$ Edition, Volume 2, Boston: Mc Graw Hill 2008, 2748.

11. Richard J. Pollack, James H. Maguire, Fauci A.S., Braunwald E., Harrison's Principles of Internal Medicine, $17^{\text {th }}$ Edition, Volume 2, Boston: Mc Graw Hill 2008, 2748.

12. Burnettee JC. Change of life in Women. B. Jain Publishers (P) Ltd, New Delhi, First edition, Reprint edition 1898.

13. Dudgeon RE. Lectures in Theory and Practice of Homoeopathy. B. Jain Publishers (P) Ltd, New Delhi, Reprint edition 1997, 299 - 300.

14. Internet source: Reddy SR. Effect of homoeopathic medicine Lycopodium clavatum in urinary calculi. International Journal of Applied Research 2017;3(1):790-1. Dated June 2, 2021. 
15. Shroyen F. Essential Synthesis, B. Jain Publishers (P) Ltd., New Delhi 2012, 1923.

16. Shroyen F. Essential Synthesis. B. Jain Publishers (P) Ltd. New Delhi 2012, 1948.

17. Shroyen F. Essential Synthesis. B. Jain Publishers (P) Ltd. New Delhi 2012, 1948.

18. Nash EB. Nash Leaders in Homoeopathic Therapeutics. Reprint edition, B. Jain Publishers (P) Ltd, New Delhi 1992.

19. Ryckman RM. Theories of Personality, $5^{\text {th }}$ edition, Califernia: Brooks/Cole Publishing Company 1993, 33 $-34$.

20. Kent JT. Lectures on Homoeopathic Philosophy. B. Jain Publishers (P) Ltd. Reprint Edition 1997, 137. 